\title{
pnmbalina
}

(8)

\section{Evaluación del riesgo de inundaciones mediante tecnología de geo-procesamiento raster y vectorial}
Autor(es):
Andrade, María Isabel; Schomwandt, David; Lucioni, Nora
Publicado por: Imprensa da Universidade de Coimbra; RISCOS - Associação
URL persistente: Portuguesa de Riscos, Prevenção e Segurança
DOI: DOl:http://dx.doi.org/10.14195/978-989-96253-3-4_19
Accessed : $\quad$ 26-Apr-2023 13:03:02

A navegação consulta e descarregamento dos títulos inseridos nas Bibliotecas Digitais UC Digitalis, UC Pombalina e UC Impactum, pressupõem a aceitação plena e sem reservas dos Termos e Condições de Uso destas Bibliotecas Digitais, disponíveis em https://digitalis.uc.pt/pt-pt/termos.

Conforme exposto nos referidos Termos e Condições de Uso, o descarregamento de títulos de acesso restrito requer uma licença válida de autorização devendo o utilizador aceder ao(s) documento(s) a partir de um endereço de IP da instituição detentora da supramencionada licença.

Ao utilizador é apenas permitido o descarregamento para uso pessoal, pelo que o emprego do(s) título(s) descarregado(s) para outro fim, designadamente comercial, carece de autorização do respetivo autor ou editor da obra.

Na medida em que todas as obras da UC Digitalis se encontram protegidas pelo Código do Direito de Autor e Direitos Conexos e demais legislação aplicável, toda a cópia, parcial ou total, deste documento, nos casos em que é legalmente admitida, deverá conter ou fazer-se acompanhar por este aviso.

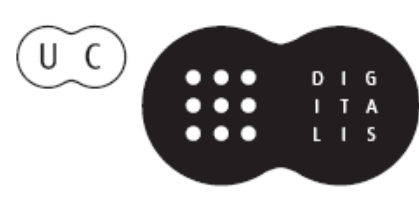



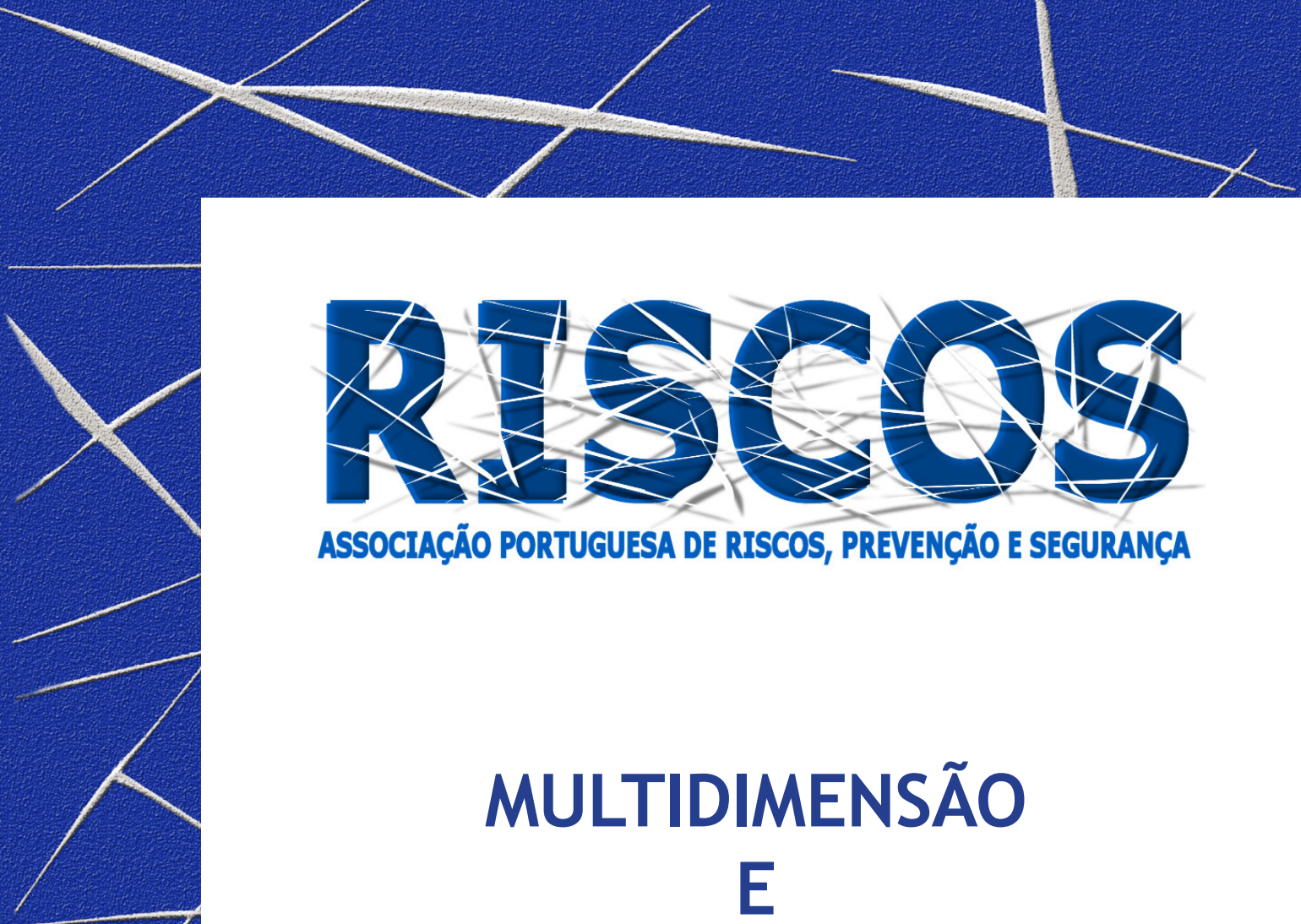

ASSOCIAÇÃO PORTUGUESA DE RISCOS, PREVENCCÃO E SEGURANÇA

MULTIDIMENSÃO

E
TERRITÓRIOS DE RISCO

III Congresso Internacional

I Simpósio Ibero-Americano

VIII Encontro Nacional de Riscos

Guimarães

2014 


\title{
EVALUACIÓN DEL RIESGO DE INUNDACIONES MEDIANTE TECNOLOGÍA DE GEO-PROCESAMIENTO RASTER Y VECTORIAL
}

\author{
María Isabel Andrade \\ Centro de Investigaciones Geográficas (CIG). IdIHCS. Facultad de Humanidades y Ciencias de Educación. \\ Universidad Nacional de La Plata (UNLP). Argentina \\ miandrade1911@gmail.com \\ David Schomwandt \\ Centro de Investigaciones Geográficas (CIG). IdIHCS. Facultad de Humanidades y Ciencias de Educación. \\ Universidad Nacional de La Plata (UNLP). Argentina \\ schomwandt@gmail.com \\ Nora Lucioni \\ Centro de Investigaciones Geográficas (CIG). IdIHCS. Facultad de Humanidades y Ciencias de Educación. \\ Universidad Nacional de La Plata (UNLP). Argentina \\ noraclucioni@gmail.com
}

\begin{abstract}
RESUMEN
El trabajo pretende exponer los primeros resultados obtenidos mediante una propuesta metodológica basada en las nuevas tecnologías de geo-procesamiento, la metodología comprende técnicas de teledetección y análisis multitemporal de tipo raster y vectorial. Los resultados bajo el análisis multivariado, fueron interpretados de acuerdo a la perspectiva de la Teoría Social del Riesgo.

El área de investigación es parte del escenario de extrema fragilidad de la llanura pampeana, gran parte de la misma ha sido sometida históricamente a eventos hidrológicos extremos, tanto de déficit como de excedentes hídricos.

Se ha logrado obtener una primera aproximación, en la caracterización y evaluación de las relaciones entre los procesos naturales y sociales que derivan de los procesos de anegabilidad. Como principal resultado se obtuvieron criterios para la evaluación de riesgo de inundación en el Gran La Plata, Región Pampeana, Argentina.

Parte de las conclusiones arribadas consideran que las modificaciones al medio natural se han llevado a cabo bajo un plan de ordenamiento territorial inadecuado. El manejo del ambiente se pone en evidencia en la salud de la población, en su calidad de vida, en los costos económicos para el mantenimiento de la cantidad y la calidad del agua para consumo y para la producción, en los costos sanitarios, económicos y sociales de la población involucrada y del Estado para hacer frente a las consecuencias de las emergencias hídricas. Palabras Clave: riesgo hídrico, vulnerabilidad, incertidumbre, SIG, sensores remotos
\end{abstract}

\section{Introducción}

El área de estudio comprende el aglomerado de Gran La Plata, integrado por los Partidos de Berisso, Ensenada y La Plata, en la Provincia de Buenos Aires, Argentina (Figura 1).

El marco de análisis de este trabajo, consiste en el tratamiento de las inundaciones desde la Teoría Social del Riesgo, según la cual se estudian los riesgos a determinados eventos "catastróficos" desde el reconocimiento que el abordaje realizado por la ciencia "normal" es insuficiente para el tratamiento de estas temáticas. Entendemos por ciencia "normal" aquella que responde al paradigma vigente. La visión reduccionista que ésta aplica deja afuera problemas importantes y/o manipula técnica o cuantitativamente variables de difícil cuantificación, como por ejemplo la incertidumbre (Funtowicz, 1994). La existencia de problemas ambientales reales, concretos, que afectan a una población real y concreta, suponen la búsqueda de herramientas metodológicas en un nuevo estilo de actividad científica que tenga en cuenta que lo "normal" puede ser la incertidumbre y la impredictibilidad. 
La recurrencia del fenómeno de inundación en el área de estudio, no se debe sólo a causas naturales, sino también a factores antrópicos. Entre los factores naturales más relevantes se destacan: el régimen de precipitaciones, topografía del terreno plana, de muy bajo gradiente de pendiente y suelos hidromorfos de muy baja permeabilidad. Entre los factores antrópicos, pueden citarse: las modificaciones en el drenaje natural, con la consecuente pérdida en la capacidad de captar y almacenar agua de lluvia de los suelos por deterioro físico; el diseño de una infraestructura vial, que condiciona la acumulación y movimiento del agua en superficie; y la construcción de canales de manera anárquica, sin un criterio integral sobre el funcionamiento hidrológico regional.
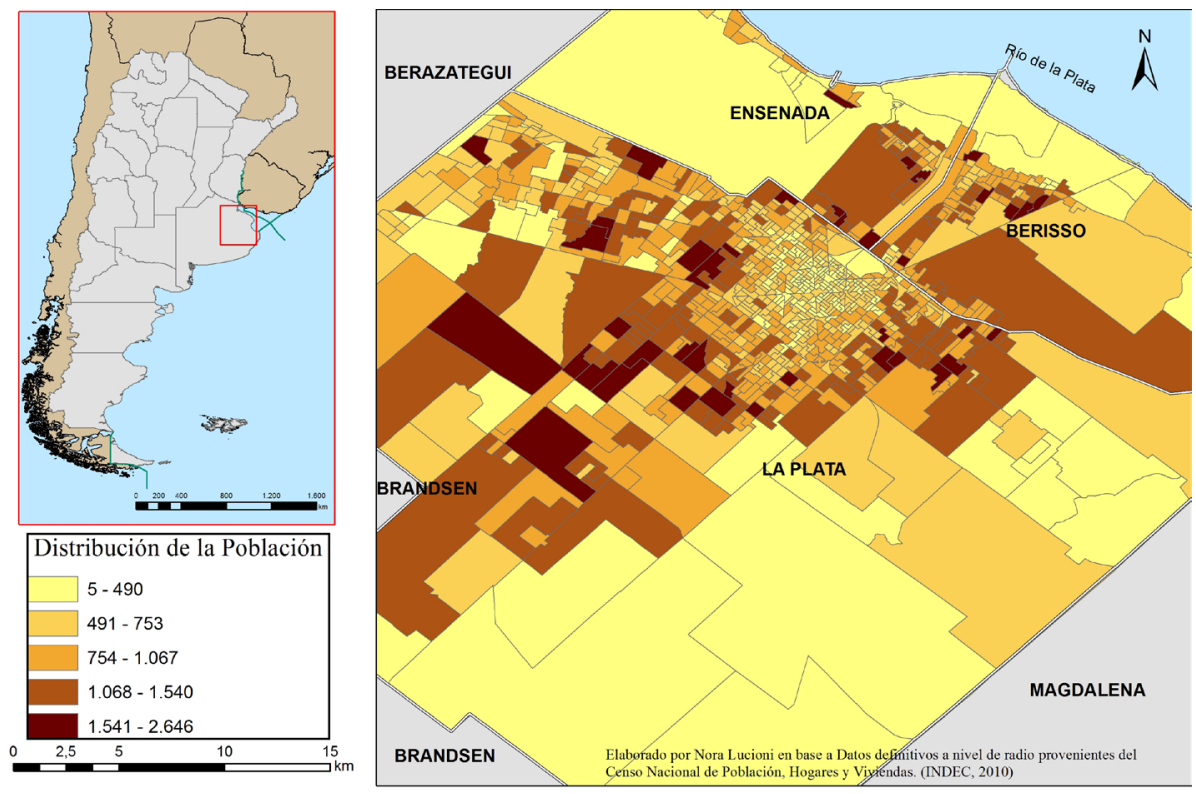

Figura 1 - Mapa de Ubicación

\section{Metodología}

Las investigaciones más recientes que consideran a la cartografía del riesgo como herramienta en el análisis de la vulnerabilidad, se han centrado en definir, cuantificar y analizar la población expuesta a amenazas de índole ambientales (Andrade, 2009). La metodología que se expone en este trabajo pretende alcanzar la expresión cartográfica como herramienta útil en la valoración y gestión del riesgo al establecer una zonificación con la ayuda de la combinación de mapas de niveles de peligrosidad, vulnerabilidad y exposición. La línea de investigación que persigue en este trabajo considera a los Sistemas de Información Geográfica (SIG) en combinación con la teledetección, una herramienta fundamental al momento de desarrollar modelos de exposición y vulnerabilidad en el área de estudio.

La construcción de una cartografía preliminar de riesgo de inundación del Gran La Plata fue a partir del tratamiento de sus componentes básicos:

- Peligrosidad es el potencial de peligro inherente a los fenómenos naturales que puede agudizarse por acciones humanas. En este trabajo se identifica la peligrosidad teniendo en 
cuenta la red hidrográfica superficial, la altimetría del área, los usos de suelos y las obras de infraestructura que afectan el escurrimiento.

- Exposición: hace referencia a la distribución territorial de las personas y bienes que pueden verse afectados ante un evento catastrófico. La exposición precisa la interrelación entre peligrosidad y vulnerabilidad. Su construcción se elabora mediante la interpretación cartográfica y teledetección de usos de suelo y población afectada, para identificar áreas críticas.

- Vulnerabilidad: es la capacidad de hacer frente a un evento catastrófico. Se haya condicionada por la situación socioeconómica previa a la ocurrencia del evento.

\section{Materiales y Métodos}

El trabajo se apoyó en información fotogramétrica proveniente del Instituto Geográfico Nacional (IGN). Un producto fue el Modelo Digital de Terreno (MDT) de resolución espacial de 7 metros, a partir de este se obtuvo el modelo de dirección del flujo (flow direction) basado en la metodología descripta por Jenson et al (1988). El segundo producto provisto por el IGN fue un mosaico fotogramétrico, en color natural e infrarrojo, de 0,40 metros de resolución espacial. Este último sirvió como base para la construcción en detalle del entramado urbano ajustado por captura de puntos GPS en terreno. Una vez registrada la información vectorial se extrajeron los valores de altura del MDT y los valores de dirección de flujo, ambos coincidentes con las intersecciones de los ejes calles. De esta manera se excluyó la topografía edilicia, las alturas asociadas al proceso de verticalización de la ciudad y sólo se concentró en los valores de cotas del trazado vial urbano. Con ello se obtuvieron las variaciones topográficas y las direcciones de flujo de la totalidad del entramado urbano del Gran La Plata.

En este trabajo se han procesado los datos provenientes del último Censo Nacional de Población, Hogares y Viviendas 2010 publicados por el Instituto Nacional de Estadísticas y Censos (INDEC) con la finalidad de obtener una caracterización socioeconómica de la población con riesgo de inundación.

\section{Resultados}

En función de este abordaje técnico se construyó la cartografía preliminar de riesgo de inundación del Gran La Plata, que considera las características topográficas, la geomorfología y de una manera indirecta los factores hidrodinámicos, en esa misma línea también se considera el riesgo que combina el nivel de agua y las velocidades. De esta manera las áreas con riesgo de inundación que fueron clasificadas como "Muy Alto" riesgo de inundación, reúne el criterio de áreas de menor cota relativa, asociadas a cauces y planicies de inundación actualmente urbanizadas o en proceso de estarlo (Figura 2). Un punto más, considerado en la generación de cartografía de riesgo, fue el lapso de tiempo en el cual se mantuvo anegada una porción de la superficie del terreno, esta consideración involucra una serie de factores como es el caso de las características topográficas, que a su vez derivan en la escorrentía o dirección de flujo.

A fin de tener una aproximación del lapso de tiempo de las áreas anegadas, se utilizó teledetección como método indirecto. La imagen Landsat 8 del día 13 de abril de 2013 capturada 11 días posteriores al fenómeno de inundación en Gran La Plata sirvió como indicador de áreas anegadas. 


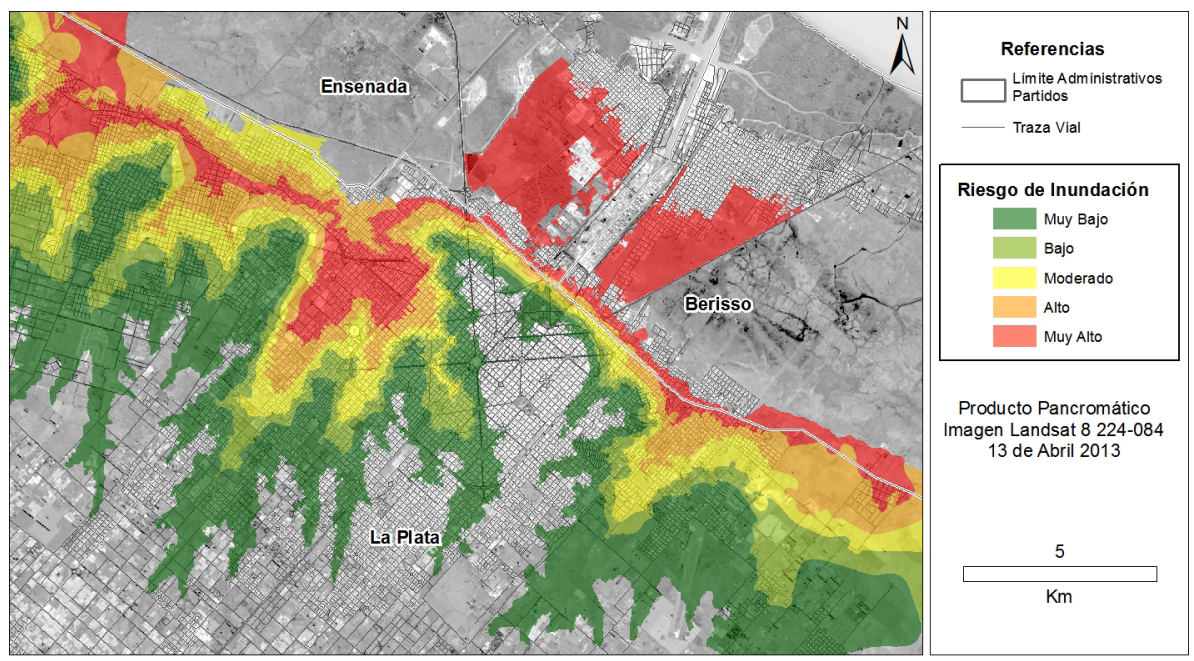

Figura 2 - Mapa de Riesgo de Inundación, Gran La Plata, Província de Buenos Aires, Argentina

Mediante la interpretación cartográfica y teledetección de usos de suelo y el procesamiento de los datos demográficos se procedió a calcular la población afectada por las áreas críticas de riesgo de inundación identificadas principalmente como “Alto” y “Muy alto” en el Gran La Plata (Tabla I).

Tabla I. Población afectada por las áreas calculadas con alto riesgo de inundación

\begin{tabular}{|c|c|c|c|c|c|}
\hline PARTIDO & $\begin{array}{c}\% \\
\text { Población }\end{array}$ & $\begin{array}{c}\% \\
\text { Hogares }\end{array}$ & $\begin{array}{c}\text { \% Hogares } \\
\text { con } \mathrm{NBI}^{*}\end{array}$ & $\begin{array}{l}\text { \%Población } \\
\text { Desocupada }\end{array}$ & Riesgo \\
\hline BERISSO & 22,49 & 21,29 & 37,63 & 24,50 & Muy alto \\
\hline ENSENADA & 21,23 & 20,53 & 21,02 & 22,77 & Muy alto \\
\hline LA PLATA & 7,16 & 7,03 & 4,87 & 7,41 & Muy alto \\
\hline PARTIDO & $\begin{array}{c}\% \\
\text { Población }\end{array}$ & $\begin{array}{c}\% \\
\text { Hogares }\end{array}$ & $\begin{array}{c}\text { \% Hogares } \\
\text { con } \mathrm{NBI}^{*}\end{array}$ & $\begin{array}{l}\text { \%oblación } \\
\text { Desocupada }\end{array}$ & Riesgo \\
\hline BERISSO & 2,70 & 3,06 & 1,91 & 2,38 & Alto \\
\hline ENSENADA & 0,24 & 0,24 & 0,25 & 0,18 & Alto \\
\hline LA PLATA & 11,06 & 10,73 & 9,53 & 11,11 & alto \\
\hline
\end{tabular}

*NBI: necesidades básicas insatisfechas

Tal como se visualiza en la Tabla, la misma arroja que un alto porcentaje de la población con elevados porcentajes de NBI y concentrados en las periferias de los núcleos urbanos son susceptibles de ser afectados ante eventos extremos. Por otra parte, según investigaciones anteriores, al efectuarse la superposición entre las zonas Inundables y la Densidad de la Mancha Urbana, se determinó que en el casco fundacional, donde se encuentra la mayor heterogeneidad de densidad, se observan las zonas en donde predominan las áreas caracterizadas con valores de alto riesgo (Andrade et al, 2012).

\section{Conclusión}

La metodología aportó información sobre la modificación del sistema de drenaje natural debido al proceso de la urbanización, esto a su vez derivó en una primera aproximación de la cartografía en detalle de las áreas de riesgo de inundación en el Gran La Plata. 
Se prevé en un futuro, la realización del análisis de las relaciones existentes entre los cambios producidos por el proceso de urbanización sobre la cuenca y la ocurrencia de los eventos de inundación a fin de cuantificar las áreas expuestas a ser anegadas o inundadas. En este sentido, se pretende realizar modelos predictivos para evaluar el riesgo hídrico en base a datos de percepción remota, estudios multitemporales y complementados con trabajo de campo de los sectores de peligrosidad natural ocupados por la expansión urbana en la actualidad y considerando las posibles expansiones urbanas en un futuro cercano.

Un aporte que esta investigación pretende llegar hacer a futuro es colaborar con la cartografía de gestión de emergencias, la misma es de fuerte carácter aplicativo por cuanto que su finalidad es la utilización en los procesos operativos de intervención ante la ocurrencia de desastres naturales.

\section{Bibliografía}

Andrade, M. I. (2009) “Aplicación de la TD en la obtención de datos para mitigar la incertidumbre en el riesgo hídrico. Fenómenos de inundaciones y sequías en Buenos Aires, Argentina”. En: Teledetección. Agua y Desarrollo Sostenible. Editores: Salomón Montesinos Aranda y Lara Fernández Fornos. ISBN 978-84613-4257-0. Calatayud. Septiembre de 2009.

Andrade, M.I., Lucioni, N. e lezzi, L. (2012) “Factores de riesgo de inundaciones en el Gran La Plata, Argentina”. En: IX Jornadas Nacionales de Geografía Física. Departamento de Geografía y Turismo de la Universidad Nacional del Sur, Bahía Blanca, del 19 al 21 de abril de 2012. 14-24 pp. ISBN 978-9871648-32-0

INDEC (2010) Censo Nacional de Población, Hogares y Viviendas 2010. Datos definitivos a nivel de radios censales. Argentina.

Jenson S. K., J. O. Dominguez. (1988) “Extracting Topographic Structure from Digital Elevation Data for Geographic Information System Analysis”. En: Photogrammetric Engineering and Remote Sensing. Vol. $54, N^{\circ} 11$, november 1988, pp. 1593-1600.

Funtowicz, S. (1994) “Epistemología política. Ciencia con la gente”. En: Serie Documentos e Informes de Investigación N 187, Mayo 1994, FLACSO, Buenos Ares, Argentina. 